\title{
Berberine protects against lipopolysaccharide- induced intestinal injury in mice via alpha 2 adrenoceptor-independent mechanisms
}

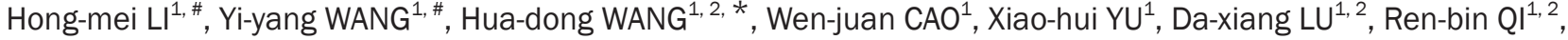 \\ Chao-feng $\mathrm{HU}^{1}$, Yu-xia YAN ${ }^{3}$ \\ ${ }^{1}$ Department of Pathophysiology, Key Laboratory of State Administration of Traditional Chinese Medicine, School of Medicine, Ji-nan \\ University, Guangzhou 510632, China; ${ }^{2}$ Institute of Integrative Medicine, Ji-nan University, Guangzhou 510632, China; ${ }^{3}$ Department of \\ Biochemistry, School of Medicine, Ji-nan University, Guangzhou 510632, China
}

\begin{abstract}
Aim: To investigate the mechanisms responsible for the protective action of berberine (Ber) against gut damage in endotoxemic mice. Methods: Male BALB/c mice were administered intragastrically with distilled water $(0.1 \mathrm{~mL} / 10 \mathrm{~g})$, Ber $(50 \mathrm{mg} / \mathrm{kg})$ alone, yohimbine $(2$ $\mathrm{mg} / \mathrm{kg}$ ) alone, or Ber $(50 \mathrm{mg} / \mathrm{kg})$ in combination with yohimbine $(2 \mathrm{mg} / \mathrm{kg}$ ) for $3 \mathrm{~d}$. On the third day, lipopolysaccharide (LPS, $18 \mathrm{mg} / \mathrm{kg})$ or normal saline was intraperitoneally injected one hour after the intragastric administration. Following the treatment, intestinal injury in the ileum was histopathologically accessed; enterocyte apoptosis was examined using TUNEL method; Toll-like receptor 4 (TLR4) mRNA expression was measured using RT-PCR assay; inhibitor protein-kB $\alpha(\mathrm{I}-\mathrm{kB} \alpha)$ phosphorylation and myeloperoxidase content were examined using Western blloting. The macrophage inflammatory protein-2 (MIP-2) production was measured using ELISA assay. Results: Mice challenged with LPS caused extensive ileum injury, including a significantly increased injury score, decreased intestinal villus height, reduced gut mucosal weight and increased intestinal permeability. Furthermore, LPS significantly induced enterocyte apoptosis, increased TLR4 mRNA expression, I-kB $\alpha$ phosphorylation, MIP-2 production and myeloperoxidase content in the ileum. Pretreatment with Ber significantly alleviated all the alterations in the ileum in the endotoxemic mice. Pretreatment with the $\alpha 2$-adrenoceptor antagonist yohimbine did not block the protective action of Ber against LPS-induced intestinal injury. In addition, treatment with yohimbine alone did not prevent LPS-induced intestinal injury.

Conclusion: Pretreatment with Ber provides significant protection against LPS-induced intestinal injury in mice, via reducing enterocyte apoptosis, inhibiting the TLR4-nuclear factor KB-MIP-2 pathway and decreasing neutrophil infiltration that are independent of $\alpha 2$-adrenoceptors.
\end{abstract}

Keywords: berberine; lipopolysaccharide; intestinal injury; apoptosis; Toll-like receptor 4 (TLR4); NF-KB; macrophage inflammatory protein-2 (MIP-2); $\alpha_{2}$-adrenoceptor; yohimbine

Acta Pharmacologica Sinica (2011) 32: 1364-1372; doi: 10.1038/aps.2011.102; published online 3 Oct 2011

\section{Introduction}

Sepsis is one of the principal causes of death in intensive care units. There are 750000 new cases of severe sepsis in the United States every year, and the incidence of sepsis is expected to continue to grow at a rate of $1.5 \%$ per year ${ }^{[1]}$. Although the exact mechanisms responsible for sepsis remain unclear, gut dysfunction has been identified in sepsis, and increased intestinal permeability is associated with the development of multiple organ dysfunction syndrome ${ }^{[2,3]}$. Indeed,

\footnotetext{
\# The first two authors contributed equally to this paper.

* To whom correspondence should be addressed.

E-mail owanghd@jnu.edu.cn

Received 2011-03-18 Accepted 2011-07-05
}

the intestinal epithelium, which is constantly exposed to bacterial products, is the first line of defense against microorganisms. An intact and functioning mucosal barrier, maintained by the intestinal epithelial cells, is crucial to prevent potentially pathogenic bacteria in the intestinal lumen from translocating to the normally sterile compartments of the body ${ }^{[4]}$. Therefore, it is critical to protect gut barrier function when providing treatment for sepsis.

Lipopolysaccharide (LPS), a major component of the outer membrane of Gram-negative bacteria, is recognized as the most potent microbial mediator in the pathogenesis of sepsis ${ }^{[5]}$. Extensive studies in experimental model systems have established that LPS challenge impairs the integrity of the intestinal $\operatorname{mucosa}^{[6,7]}$. 
Berberine (Ber) is an isoquinoline alkaloid found in Coptidis rhizoma. It is well known that Ber has many biological activities, such as the regulation of inflammation ${ }^{[8]}$. Recently, we and others have shown that pretreatment with Ber protects against intestinal injury in LPS-challenged animals ${ }^{[9,10]}$. However, the mechanisms involved in the protective effect of Ber on LPS-induced gut injury are not fully understood. Ber has also been reported to activate the $a_{2}$-adrenoceptor ${ }^{[11]}$. We have also shown that blockage of the $\mathrm{a}_{2}$-adrenoceptor with yohimbine $(Y, 2 \mathrm{mg} / \mathrm{kg}$ ) enhances the protective effect of Ber (50 mg/ kg) against LPS-induced lethality and that the combination of Ber and $\mathrm{Y}$ improves survival in mice subjected to cecal ligation and puncture ${ }^{[12]}$. In this context, the role of the $\mathrm{a}_{2}$-adrenoceptor in the protection of Ber against LPS-induced intestinal injury needs to be examined.

Several mechanisms have been shown to be responsible for LPS-induced intestinal injury. These include the activation of the Toll-like receptor 4 (TLR4)-nuclear factor-kB (NF-kB) signaling pathway, expression of macrophage inflammatory protein-2 (MIP-2), and tissue infiltration by neutrophils and apoptosis of enterocytes ${ }^{[7,13-17]}$. Therefore, the purpose of this study was to investigate the effect of Ber on the TLR4-NF-kB signaling pathway, MIP-2 expression, neutrophil recruitment and enterocyte apoptosis in the ileum of LPS-treated mice and, furthermore, to determine the role of the $a_{2}$-adrenoceptor in this process.

\section{Materials and methods \\ Animals}

Male BALB/c mice (6-8 weeks, weighing 21-23 g) were purchased from Guangdong Medical Laboratory Animal Center (Guangzhou, China). Animals were kept at $24 \pm 2{ }^{\circ} \mathrm{C}$ and exposed to a twelve hours light/dark cycle for a minimum of $3 \mathrm{~d}$ before the experiment, during which time they had free access to water and mouse chow. All experiments were conducted in compliance with the Guide for the Care and Use of Laboratory Animals published by the US National Institutes of Health (NIH Publication № 85-23, revised 1996) and were approved by the Animal Care and Use Committee at School of Medicine, Ji-nan University.

\section{Experimental design}

Male BALB/c mice were randomly divided into 8 groups: control, LPS, Ber+LPS, Ber+Y (an $\mathrm{a}_{2}$ adrenoceptor antagonist)+LPS, Y+LPS, Ber, Ber+Y, and Y. Once a day for 3 days, the mice were treated intragastrically with the following: distilled water $(0.1 \mathrm{~mL} / 10 \mathrm{~g})$, Ber $(50 \mathrm{mg} / \mathrm{kg})$, Ber $(50 \mathrm{mg} / \mathrm{kg})$ in combination with $Y(2 \mathrm{mg} / \mathrm{kg})$ or $Y(2 \mathrm{mg} / \mathrm{kg})$. One hour after last intragastric treatment, LPS $(18 \mathrm{mg} / \mathrm{kg}$, derived from Escherichia coli O55:B5) or normal saline was injected intraperitoneally. Separate experiments were performed for each measurement. Neutral sulfate Ber, Y, and LPS were purchased from Sigma Chemical Co (St Louis, MO, USA).

\section{Histopathological examination}

The animals were anesthetized and sacrificed twelve hours after the injection of LPS or normal saline, and $1 \mathrm{~cm}$ of ileum tissue, beginning at $1 \mathrm{~cm}$ proximal to the ileocecal junction, was removed and fixed in a $4 \%$ formaldehyde solution. The fixed intestinal tissues were embedded in paraffin wax using standard techniques. Slices $(5 \mu \mathrm{m})$ were cut and stained with hematoxylin and eosin and were examined under a light microscope. For the histological assessment of intestinal injury, a $0-4$ grading scale was used as follows ${ }^{[18]}$ : $0=$ normal histology; $1=$ slight disruption of the surface epithelium; $2=$ epithelial cell loss injury at villus tip; $3=$ mucosal vasocongestion, hemorrhage, and focal necrosis with loss of less than one-half of the villi; and 4=damage extending to more than one-half of the villi. An observer who was unaware of the treatment performed all assessments of damage. The final score was expressed as a mean rank.

The height of each complete villus in one randomly picked microscopic field $(\times 100)$ was determined by measuring the distance from the crypt-villus junction to the villus tip ${ }^{[19]}$.

\section{Intestinal mucosal weight measurement}

The animals were anesthetized and sacrificed twelve hours after the injection of LPS or normal saline. The ileum segment $(10 \mathrm{~cm})$, beginning $1 \mathrm{~cm}$ proximal to the ileocecal junction, was excised, cut longitudinally, washed with cold isotonic saline and weighed (W1). After the mucosa was scraped off using a glass slide ${ }^{[6,7]}$, the ileum tissue was weighed again (W2). The weight of the mucosa was calculated as the difference between $\mathrm{W} 1$ and $\mathrm{W} 2$ (W=W1-W2), and the result was expressed as mg per centimeter of ileum length per $10 \mathrm{~g}$ of body weight.

\section{Intestinal permeability assessment}

Intestinal permeability was assessed using a slightly modified version of the method described by Yasuda et $a l^{[20]}$. Mice were anesthetized and sacrificed twelve hours after the challenge with LPS or normal saline. A $15-\mathrm{cm}$ segment of ileum was dissected beginning $1 \mathrm{~cm}$ proximal to the ileocecal junction. The intestinal lumen was gently washed with ice-cold PBS (0.01 $\mathrm{mol} / \mathrm{L}, \mathrm{pH} 7.4$ ), and one side of intestine was ligated with a 4-0 silk suture. Fluorescein isothiocyanate (FITC)-dextran powder (FD4, MW 4 kDa, Sigma, St Louis, MO, USA) was dissolved in PBS $(0.01 \mathrm{~mol} / \mathrm{L}, \mathrm{pH}$ 7.4) to a final concentration of $4 \mathrm{mg} / \mathrm{mL}$. FITC-dextran solution $(0.5 \mathrm{~mL})$ was applied to the intestinal lumen that had a well-protected intestinal wall. Next, the other side of intestine was ligated. The outside of the intestinal pouch was washed and gently shaken in $3 \mathrm{~mL}$ PBS at $37^{\circ} \mathrm{C}$ for $60 \mathrm{~min}$. The intestinal permeability of the pouch was evaluated ex vivo by measuring the amount of FITC-dextran that leaked out of the intestinal pouch. A standard solution was prepared as follows: FITC-dextran powder was diluted at different concentrations $(0,1.56 \mu \mathrm{g} / \mathrm{mL}, 3.13 \mu \mathrm{g} / \mathrm{mL}, 6.25$ $\mu \mathrm{g} / \mathrm{mL}, 12.5 \mu \mathrm{g} / \mathrm{mL}, 25 \mu \mathrm{g} / \mathrm{mL}, 50 \mu \mathrm{g} / \mathrm{mL}, 100 \mu \mathrm{g} / \mathrm{mL})$ with PBS $(0.01 \mathrm{moL} / \mathrm{L}, \mathrm{pH} 7.4)^{[21]}$. Sample supernatants $(200 \mu \mathrm{L})$ and standard solutions were pipetted into duplicate wells of a black microtiter plate, and the fluorescence was measured at an excitation wavelength of $485 \mathrm{~nm}$ and an emission wavelength of $520 \mathrm{~nm}$. 


\section{Apoptotic cell evaluation}

The terminal deoxyuridine nick end-labeling (TUNEL) immunohistochemical method (ApopTag in situ Apoptosis Detection Kit, Roche Co, Basel, Switzerland) was used to identify and quantitate apoptotic cells in the ileum. Briefly, after deparaffinization, the sections were treated with $20 \mu \mathrm{g} / \mathrm{mL}$ proteinase $\mathrm{K}$ for $15 \mathrm{~min}$ at room temperature and with $0.3 \%$ $\mathrm{H}_{2} \mathrm{O}_{2}$ for 10 min. Following incubation with the equilibration buffer for $10 \mathrm{~min}$ at room temperature, the sections were incubated with terminal deoxynucleotidyl transferase enzyme reaction mixture for $60 \mathrm{~min}$ at $37^{\circ} \mathrm{C}$. Further incubation with peroxidase-conjugated antibody was performed for $30 \mathrm{~min}$ at $37^{\circ} \mathrm{C}$. The sections were stained with diaminobenzidine solution and counterstained with hematoxylin. TUNELpositive cells, identified by the brown staining of the nucleus and their apoptotic morphology ${ }^{[19,22]}$, were counted by an observer, blinded to the experimental conditions, who selected 10 full-length villi in each sample. The apoptosis index (AI) of enterocytes was calculated as the ratio of the total number of TUNEL-positive cells to the total number of epithelial cells $\times 100 \%{ }^{[19]}$.

\section{Myeloperoxidase activity assay}

Mice were challenged with LPS or normal saline, and twelve hours later, ileum tissue, beginning $1 \mathrm{~cm}$ proximal to the ileocecal junction, was removed, washed with cold isotonic saline and homogenized thoroughly in a homogenate solution. Myeloperoxidase (MPO) activity was determined by a spectrophotometric method using myeloperoxidase activity detection kits (Nanjing Jiancheng Bioengineering Institute, Nanjing, China) according to the manufacturer's specifications.

\section{MIP-2 protein analysis}

Ileum tissue was collected two hours after intraperitoneal injection of LPS or normal saline, thoroughly homogenated in an isotonic sodium chloride solution and centrifuged at $3000 \times g$ for $10 \mathrm{~min}\left(4^{\circ} \mathrm{C}\right)$. The supernatant was collected, and the concentration of MIP-2 was determined using mouse MIP-2 enzyme-linked immunosorbent assay kits (R\&D Systems, Minneapolis, MN, USA). The total protein level was measured using a commercially available bicinchoninic acid protein assay kit (ShennengBocai Co, Shanghai, China).

\section{Western blotting}

The mice were killed twelve hours after LPS or normal saline injection. Ileum tissues were removed, washed with cold normal saline and thoroughly homogenated on ice in RIPA lysis buffer (Bioteke Co, Beijing, China) that was supplemented with the protease inhibitor phenylmethanesulfonyl fluoride. The lysates were centrifuged at $12000 \times g$ at $4{ }^{\circ} \mathrm{C}$ for $10 \mathrm{~min}$, and the supernatants were aliquoted into fresh tubes and stored at $-80^{\circ} \mathrm{C}$ until measurement. Protein concentrations in the lysates were determined using a bicinchoninic acid protein assay kit (ShennengBocai Co, Shanghai, China). Protein samples $(70 \mu \mathrm{g})$ were separated by sodium dodecyl sulfate polyacrylamide gel electrophoresis and transferred to a
Hybond-C pure nitrocellulose membrane. After blocking, the membranes were probed with the following antibodies: rabbit antibodies against cleaved caspase-3 (Cell Signaling Technology, Danvers, USA), inhibitor protein-kBa (I-kBa, Cell Signaling Technology, Danvers, USA), phosphorylated I-kBa (Cell Signaling Technology, Danvers, USA), or glyceraldehyde-3phosphate dehydrogenase (GAPDH, Cell Signaling Technology, Danvers, USA) or mouse antibodies against MPO (R\&D Systems, Minneapolis, MN, USA). The membranes were incubated overnight at $4{ }^{\circ} \mathrm{C}$ with primary antibody and were later incubated with secondary antibody (peroxidase-conjugated goat anti-rabbit or anti-mice antibodies, Dingguo Changsheng Co, Beijing, China) at room temperature for one hour. The antigen-antibody complexes were visualized using a chemiluminescence system (Pierce Biotechnology Inc, Rockford, USA) and exposed to film (Kodak X-OMAT, Guangzhou, China). The relative densities of the bands were analyzed using a BI2000 image analysis system (Chengdu TME Technology Co Ltd, Chengdu, China).

\section{Real-time RT-PCR analysis of TLR4 mRNA expression}

Ileum segments were quickly isolated and frozen at $-80^{\circ} \mathrm{C}$. Total RNA was extracted from the frozen tissues using Trizol reagent (TaKaRa, Bao Biotec Co, Dalian, China) and was subjected to reverse transcription using a PrimeScript ${ }^{\circledR} \mathrm{RT}$ reagent kit (TaKaRa, Bao Biotec Co, Dalian, China) according to the manufacturer's instructions. Polymerase chain reaction amplifications were performed using the $\mathrm{SYBR}^{\circledR}$ PrimeScript ${ }^{\mathrm{TM}}$ RT-PCR Kit II (TaKaRa, Bao Biotec Co, Dalian, China), and gene-specific polymerase chain reaction products were continuously measured by means of a LightCycler ${ }^{\circledR} 480$ (F Hoffmann-La Roche, Ltd, Basel, Switzerland) detection system. The PCR reaction was performed as follows: pre-denaturation at $95^{\circ} \mathrm{C}$ for $30 \mathrm{~s}$ followed by 45 cycles of $10 \mathrm{~s}$ at $95^{\circ} \mathrm{C}$, annealing at $60^{\circ} \mathrm{C}$ for $10 \mathrm{~s}$ and elongation at $72^{\circ} \mathrm{C}$ for $10 \mathrm{~s}$. The fluorescent signal was collected at $72^{\circ} \mathrm{C}$, and the melting curve procedure was carried out. The purity of the product was analyzed using a melting curve. The PCR primers were designed as follows: TLR4 (forward 5'-GCTTTCACCTCTGCCTTCAC-3' and reverse 5'-CCAACGGCTCTGAATAAAGTG-3') and GAPDH (forward 5'-TCACCACCATGGAGAAGGC-3' and reverse 5'-GCTAAGCAGTTGGTGGTGCA-3'). Every PCR experiment was carried out in triplicate. A standard curve was generated using a series of 10-fold dilutions of cDNA from the LPS group, and the amplification efficiency of each gene was calculated. It was accepted that the amplification efficiency of TLR4 and GAPDH was approximately 2.00 and that the difference between them was lower than 0.02 .

\section{Statistical analysis}

Statistical analyses were performed using SPSS 13.0 software. The data are expressed as the mean \pm SEM. Comparisons among three or more groups were made by analysis of variance (ANOVA), and the two pairs of groups were compared using an LSD test. Ranked data are presented as the mean rank, comparisons among three or more groups were made 
by a Kruskal-Wallis $H$ test, and the two pairs of groups were compared using a Nemenyi test. A $P$ value less than 0.05 was considered statistically significant.

\section{Results}

Ber inhibits LPS-induced intestinal injury, which is not reversed by $Y$

Figure 1 and Table 1 show histological changes in the ileum and the injury score of the various groups. Control mice demonstrated a normal histological architecture, and LPSchallenged mice showed extensive ileum morphological damage, including villus necrosis, infiltration of inflammatory cells and hemorrhage. There was a significant increase in the intestinal injury score compared to control animals $(P<0.01)$. Pretreatment with Ber or Ber in combination with $\mathrm{Y}$ alleviated the intestinal damage and led to a significant decrease in the injury score in the LPS-challenged mice. In contrast, there was no significant difference in histological changes in the ileum between Ber+LPS and Ber+Y+LPS groups, and Y alone did not attenuate the intestinal injury caused by LPS $(P>0.05)$.
Table 1. Effects of berberine (Ber) or/and yohimbine $(Y)$ on the mean rank of intestinal injury score in mice twelve hours after challenge with LPS. ${ }^{\mathrm{c}} P<0.01$ vs control group. ${ }^{\mathrm{d}} P>0.05,{ }^{\mathrm{e}} P<0.05,{ }^{\mathrm{f}} P<0.01$ vs LPS group.

\begin{tabular}{lrc}
\hline Group & $n$ & Mean rank \\
\hline Control & 14 & 23.21 \\
LPS & 10 & $71.90^{\circ}$ \\
Ber+LPS & 10 & $56.70^{f}$ \\
Ber+Y+LPS & 10 & $59.90^{\mathrm{e}}$ \\
Y+LPS & 10 & $73.50^{\mathrm{d}}$ \\
Ber & 14 & 23.21 \\
Ber+Y & 8 & 22.75 \\
Y & 10 & 28.90 \\
\hline
\end{tabular}

As shown in Figure 2, the villus height (Figure 2A) and mucosal weight (Figure 2B) of the ileum was measured twelve hours after the injection of mice with LPS or normal saline. Compared to control mice, LPS-challenged mice exhibited a
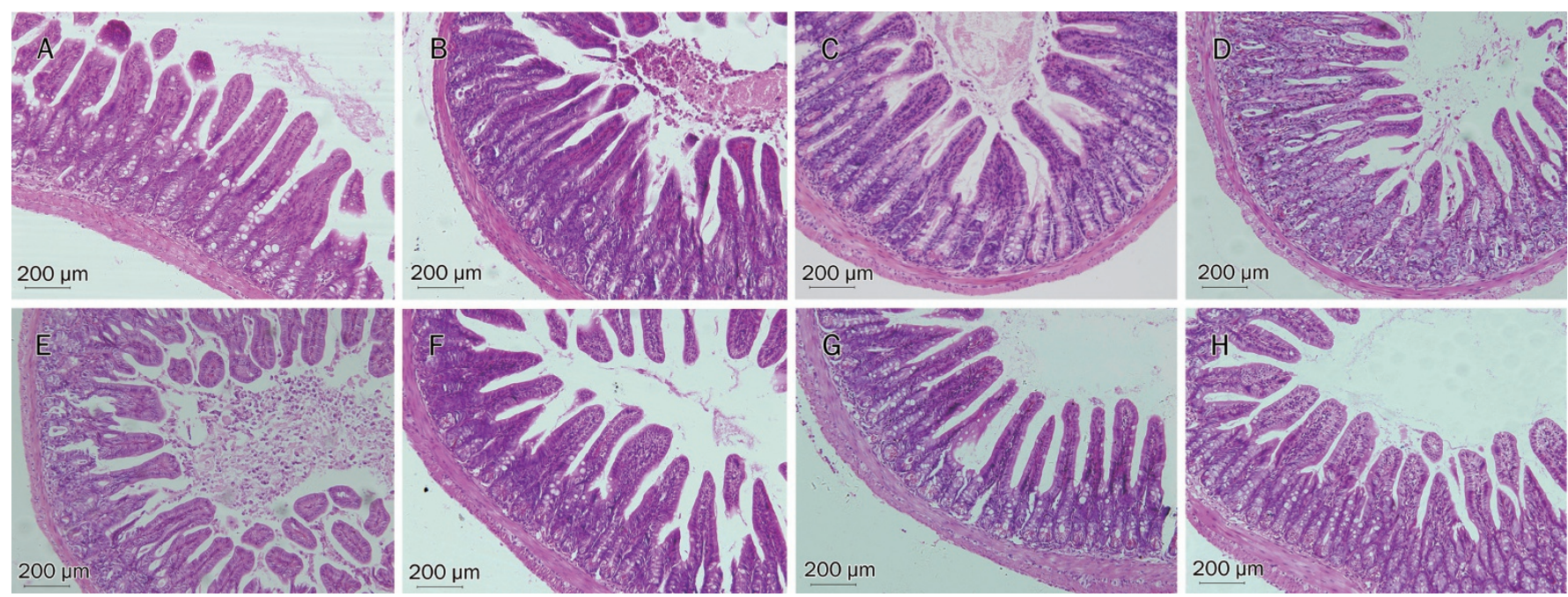

Figure 1. Photomicrograph of ileum from mice twelve hours after LPS (18 mg/kg) or normal saline injection in control (A), LPS (B), Ber+LPS (C), Ber+Y+LPS (D), Y+LPS $(E)$, Ber $(F), B e r+Y(G)$, and $Y(H)$ groups. The intestine histological sections were stained with hematoxylin-eosin. Ber: berberine; $Y$ : yohimbine. Scale bars represent $200 \mu \mathrm{m}$.
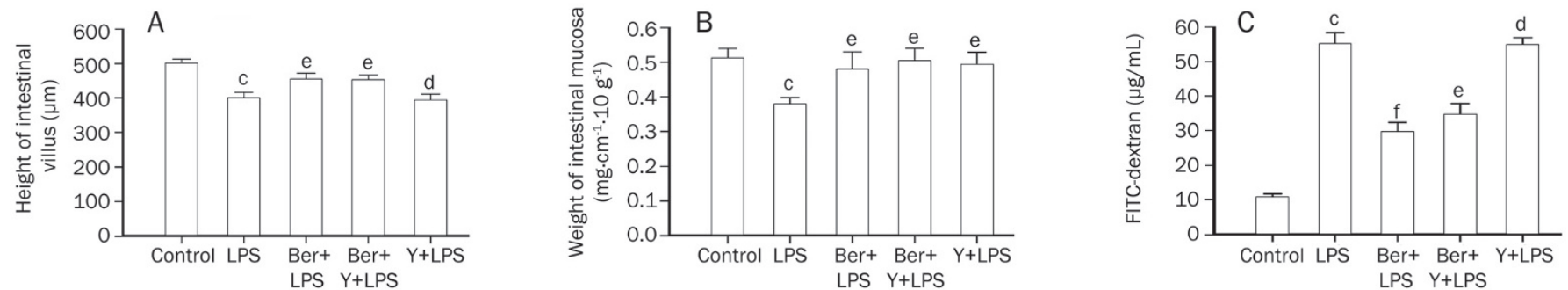

Figure 2. Effects of berberine (Ber) or/and yohimbine $(Y)$ on intestinal villus height (A), mucosa weight (B) and intestinal permeability (C) in ileum of mice twelve hours after LPS challenge $\left(18 \mathrm{mg} / \mathrm{kg}\right.$ ). Values are mean \pm SEM. $n=8-14 .{ }^{\mathrm{c}} P<0.01$ vs control group. ${ }^{\mathrm{d}} P>0.05,{ }^{\mathrm{e}} P<0.05,{ }^{\mathrm{f}} P<0.01$ vs LPS group. 
marked decrease in villus height and mucosal weight in the ileum $(P<0.01)$. The pretreatment of LPS-challenged mice with Ber or Ber in combination with $Y$ resulted in a significant increase in villus height and mucosal weight compared to the LPS-treated group $(P<0.05)$, and there was no remarkable difference in villus height or mucosal weight between Ber+LPS and Ber+Y+LPS groups. Y alone did not significantly change the villus height in LPS-challenged mice $(P>0.05)$.

The gut mucosal permeability was evaluated ex vivo by measuring the leakage of FITC-dextran from the intestinal pouch. As shown in Figure 2C, compared to control mice, an increased permeability of the ileum was observed in LPS-challenged mice twelve hours after LPS injection. Pretreatment with Ber or Ber in combination with Y significantly inhibited the LPS-induced increase in ileal mucosal permeability, and no significant difference was observed in intestinal mucosal permeability between Ber+LPS and Ber+Y+LPS groups. In contrast, $\mathrm{Y}$ alone did not significantly decrease the ileal mucosal permeability in LPS-challenged mice $(P>0.05)$. Moreover, pretreatment with Ber or $Y$ alone or Ber and $Y$ together had no significant effect on the villus height, mucosal weight or intestinal permeability of normal mice (Table 2).

Table 2. Effects of berberine (Ber) or/and yohimbine $(Y)$ on the intestine parameters of normal mice. Mean \pm SEM. $n=8-14$. ${ }^{a} P>0.05$ vs control group.

\begin{tabular}{lccc}
\hline Group & $\begin{array}{c}\text { Villus height } \\
(\mu \mathrm{m})\end{array}$ & $\begin{array}{c}\text { Mucosa weight } \\
\left(\mathrm{mg} \cdot \mathrm{cm}^{-1} \cdot \mathrm{g}^{-1}\right)\end{array}$ & $\begin{array}{c}\text { FITC-dextran } \\
(\mu \mathrm{g} / \mathrm{mL})\end{array}$ \\
\hline Control & $500.89 \pm 12.32$ & $0.51 \pm 0.03$ & $10.62 \pm 1.11$ \\
Ber & $477.40 \pm 12.00^{\mathrm{a}}$ & $0.45 \pm 0.02^{\mathrm{a}}$ & $12.00 \pm 1.09^{\mathrm{a}}$ \\
Ber+Y & $472.17 \pm 35.05^{\mathrm{a}}$ & $0.55 \pm 0.04^{\mathrm{a}}$ & $12.70 \pm 0.84^{\mathrm{a}}$ \\
$\mathrm{Y}$ & $456.96 \pm 15.99^{\mathrm{a}}$ & $0.43 \pm 0.02^{\mathrm{a}}$ & $9.02 \pm 1.02^{\mathrm{a}}$ \\
\hline
\end{tabular}

\section{Ber protects enterocyte against apoptosis induced by LPS, and $Y$ does not abolish the anti-apoptosis action of Ber}

TUNEL assays were performed to identify apoptotic cells in the ileum twelve hours after the administration of normal saline or LPS (Figure 3A-3E). Compared to control mice, a significant increase in enterocyte apoptosis in the ileum was observed after LPS challenge (Figure 3B, 4A). Following pretreatment with Ber or Ber in combination with Y, LPSchallenged mice showed a marked decrease in the $\mathrm{AI}$ in the ileum compared to the LPS-challenged animals (Figure 3B-3D, $4 \mathrm{~A})$. There was no significant difference in the AI in the ileum of LPS-treated mice following treatment with Ber or Ber in combination with $\mathrm{Y}$. In addition, treatment with $\mathrm{Y}$ alone did not change the AI in the ileum of LPS-challenged mice (Figure 3E, 4A). Western blot analysis demonstrated that there was a significant increase in the amount of cleaved caspase- 3 protein, an activated caspase-3, in the ileum of LPS-challenged mice compared to control animals (Figure 4B). Pretreatment with Ber or Ber in combination with Y markedly inhibited the
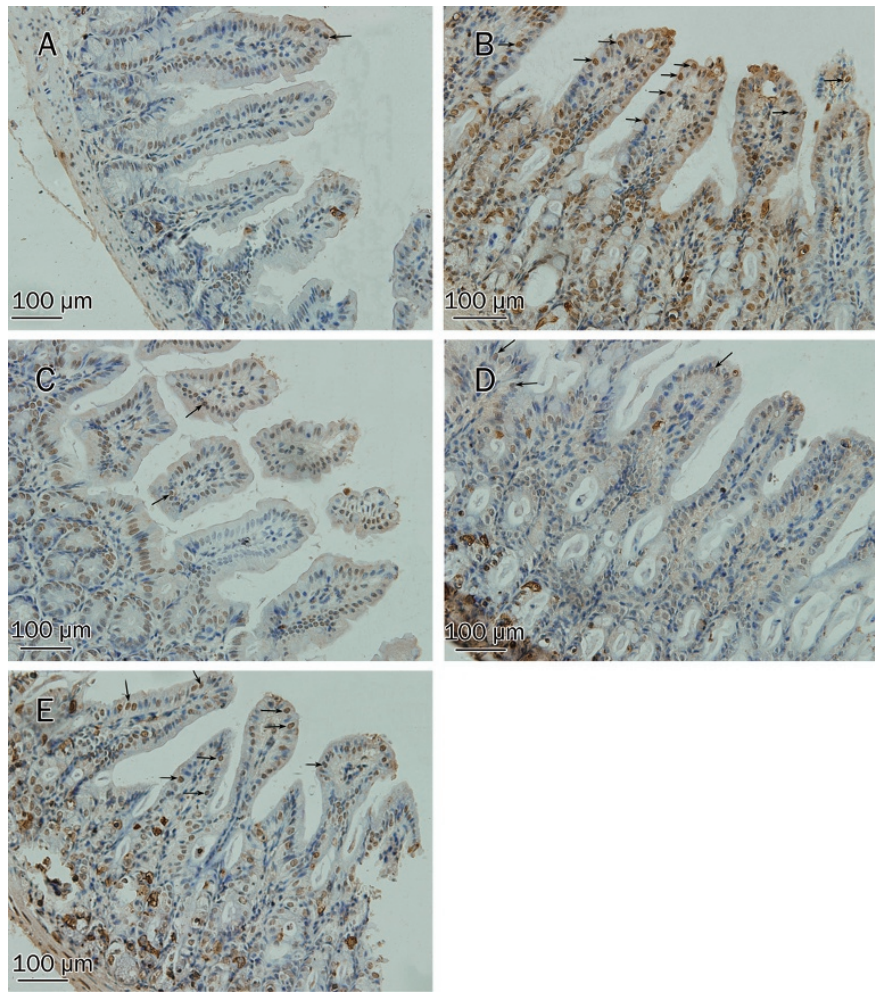

Figure 3. TUNEL analysis for enterocyte apoptosis in ileum of mice twelve hours after intraperitoneal injection of normal saline or LPS in control (A), LPS (B), Ber+LPS (C), Ber+Y+LPS (D), and Y+LPS (E) groups. TUNELpositive cells were identified as those with brown staining of the nucleus and apoptotic morphology (arrows). Scale bars represent $100 \mu \mathrm{m}$.

activation of caspase-3 in LPS-treated mice, and there was no significant difference between the two groups for the levels of cleaved caspase-3 protein in the ileum (Figure 4B). Moreover, $\mathrm{Y}$ alone did not inhibit the LPS-induced caspase-3 activation in the ileum (Figure 4B).

$Y$ does not antagonize the inhibitory effect of Ber on neutrophil infiltration in the ileum of endotoxemic mice

To evaluate the effects of Ber and Y on LPS-induced neutrophil recruitment in the ileum, MPO activity, an index of neutrophil infiltration ${ }^{[23]}$, was determined by spectrophotometric methods (Figure 5A). Compared to control mice, MPO activity was significantly increased in the ileum of LPS-challenged mice twelve hours after injection $(P<0.05)$. Pretreatment with Ber or Ber in combination with $Y$ significantly reduced MPO activity in the ileum of LPS-challenged mice $(P<0.01)$, whereas $\mathrm{Y}$ alone did not $(P>0.05)$. However, the MPO activity in the Ber control group was lower than that in the normal control $(P<0.05)$. Therefore, we measured the MPO content of the ileum using Western blotting. As shown in Figure 5B, there was no significant difference in the content of MPO in the ileum between the Ber control group and the normal control group $(P>0.05)$. Compared to control mice, MPO content was significantly increased in the ileum of LPS-challenged mice twelve hours after injection $(P<0.05)$. Pretreatment with Ber or 
A

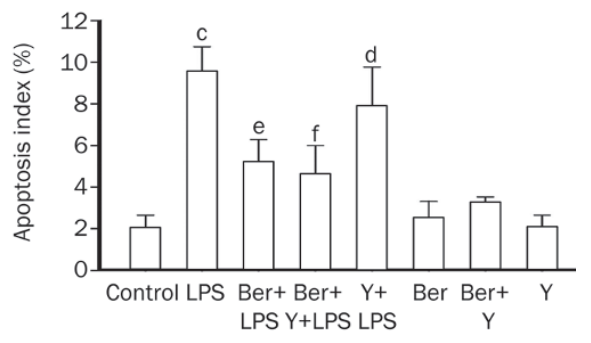

B
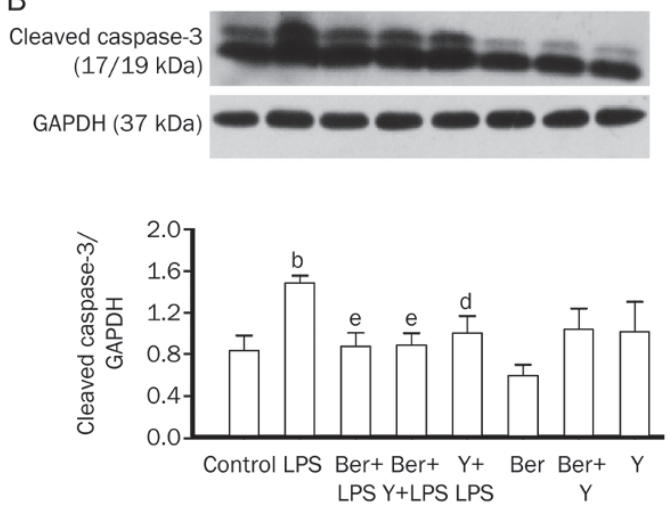

Figure 4. Yohimbine $(Y)$ did not block the inhibitory effect of berberine (Ber) on enterocyte apoptosis of ileum of mice challenged with LPS. (A) Apoptosis index (Al) was calculated as the total number of TUNEL-positive cells /the total number of epithelial cells of 10 villi. $n=9-10$. (B) Western blotting analysis for cleaved caspase-3 protein level in ileum $2 \mathrm{~h}$ after stimulation of LPS. The upper row: cleaved caspase-3 protein expression. The middle row: GAPDH protein expression. Equal amounts of proteins were loaded. The lower row: column diagrams represent the ratio of cleaved caspase-3 to GAPDH. $n=6$. ${ }^{b} P<0.05,{ }^{c} P<0.01$ vs control group. ${ }^{\mathrm{d}} P>0.05,{ }^{\mathrm{e}} P<0.05,{ }^{\mathrm{f}} P<0.01$ vs LPS group.

Ber in combination with $\mathrm{Y}$ significantly reduced MPO content in the ileum of LPS-challenged mice $(P<0.01)$, whereas $\mathrm{Y}$ alone did not $(P>0.05)$.

Ber inhibits TLR4 mRNA expression, NF-KB activation and MIP-2 production in the ileum of endotoxemic mice and this inhibition is not blocked by $Y$

As shown in Figure 6, a significant increase in TLR-4 mRNA expression, NF-KB activation and MIP-2 production was observed in the ileum of LPS-challenged mice compared to control mice. Pretreatment with Ber or Ber in combination with $\mathrm{Y}$, but not with $\mathrm{Y}$ alone, significantly decreased the LPS-induced TLR-4 mRNA expression, NF- $\mathrm{kB}$ activation and MIP-2 production in the ileum. Following the injection of normal saline, no significant difference in TLR-4 mRNA expression and NF-kB activation was observed in the ileum of control, Ber, Ber+Y, and $Y$ control groups $(P>0.05)$, and MIP-2 production in the ileum of control, Ber, Ber+Y, and $Y$ groups was undetectable.

\section{Discussion}

It has been demonstrated that Ber not only protects
A

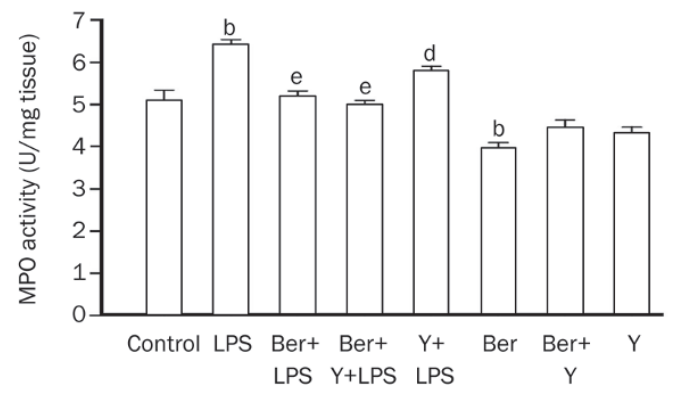

B
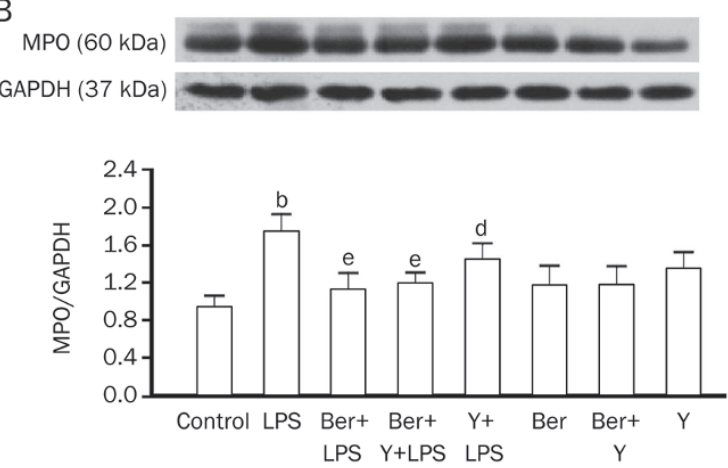

Figure 5. Yohimbine $(Y)$ did not abolish the inhibitory effect of berberine (Ber) on neutrophil infiltration in ileum of mice twelve hours after LPS (18 mg/kg) challenge. (A) Colorimetric method was used to detect myeloperoxidase (MPO) activity. $n=9-12$. (B) Western blotting analysis for MPO content and column diagrams represent mean \pm SEM for the ratio of MPO to GAPDH. $n=6 .{ }^{\mathrm{b}} P<0.05$ vs control group. ${ }^{\mathrm{d}} P>0.05,{ }^{\mathrm{e}} P<0.05$ vs LPS group.

against LPS-induced intestinal injury but also activates the $\mathrm{a}_{2}$-adrenoceptor ${ }^{[9,11]}$. Our previous studies have shown that pretreatment with $50 \mathrm{mg} / \mathrm{kg}$ Ber once a day for three days significantly improves the survival rate of LPS-challenged mice, and similar pretreatment with $2 \mathrm{mg} / \mathrm{kg} \mathrm{Y}$, an $\mathrm{a}_{2}$-adrenoceptor antagonist, in addition to Ber, enhances the protective effects of Ber in endotoxemic mice ${ }^{[9,12]}$. Therefore, it was necessary to investigate whether $\mathrm{Y}$ enhances the protective effects of Ber against LPS-induced injury in the gut.

In this study, we demonstrated that LPS challenge induced intestinal injury in mice, as evidenced by high gut permeability, decreased mucosal weight and typical histological changes, such as necrosis of the villus, infiltration by inflammatory cells and a decrease in villus height. Pretreatment with Ber protects against intestinal injury that is induced by LPS, but Y did not block the protective effect of Ber on intestinal injury in endotoxemic mice. In addition, pretreatment with Y alone did not protect against LPS-induced gut injury, as evidenced by the increase in gut permeability and the histological changes. These data indicate that pretreatment with Ber reduced LPS-induced intestinal injury independent of $\mathrm{a}_{2}$-adrenoceptor activation. However, the data from this study also show that $Y$ increases the intestinal mucosal weight of the ileum in LPS-challenged mice, and the reason for this 


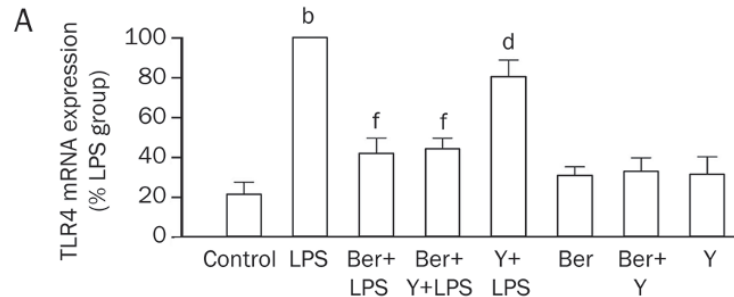

B
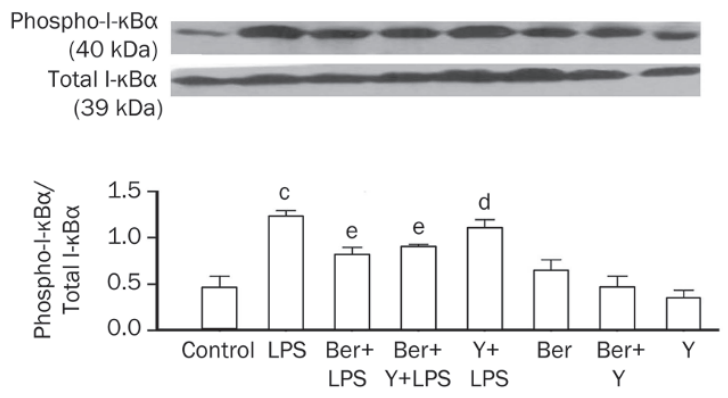

C

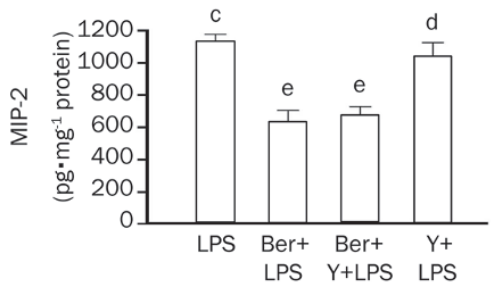

Figure 6. Yohimbine $(Y)$ did not reverse the inhibitory effect of berberine (Ber) on TLR4-NF-KB/I-KB $\alpha-M I P-2$ pathway in ileum of endotoxemic mice. (A) Real time PCR analysis for TLR4 mRNA expression in ileum $1 \mathrm{~h}$ after administration of LPS. LPS group was recognized as a control and data was expressed as the ratio to LPS group, $n=6$. (B) The total and phosphoI-kB $\alpha$ in ileum were analyzed by Western blotting $2 \mathrm{~h}$ after LPS challenge. The upper row: phospho-I-kB $\alpha$ protein expression. The middle row: total I-kB $\alpha$ protein expression. The lower row: column diagrams represent mean $\pm S E M$ for the ratio of phospho-I-kB $\alpha$ to I-kB $\alpha, n=6$. (C) ELISA analysis for MIP-2 expression in ileum twelve hours after administration of LPS. $n=10$. ${ }^{\mathrm{b}} P<0.05,{ }^{\mathrm{C}} P<0.01$ vs control group. ${ }^{\mathrm{d}} P>0.05,{ }^{\mathrm{e}} P<0.05$, ${ }^{\mathrm{f}} P<0.01$ vs LPS group.

inconsistency remains to be identified. In previous studies, we demonstrated that $\mathrm{Y}$ enhances the protective effects of Ber in endotoxemic mice ${ }^{[12]}$. In the present study, we demonstrate that $Y$ does not enhance the protective effects of Ber against intestinal damage that is caused by treatment with LPS. It has been reported that pretreatment with $\mathrm{Y}$ once a day for 3 days prevents LPS-induced myocardial dysfunction in mice ${ }^{[24]}$. Thus, the enhanced protection of Ber against endotoxemia by $Y$ may be related to the improvement of LPS-induced myocardial dysfunction in mice that are pretreated with $Y$.

It is well known that LPS challenge inhibits crypt cell proliferation, increases enterocyte apoptosis in the ileum and reduces villus height and intestinal mucosal weight. Inhibition of enterocyte apoptosis can improve intestinal recovery after LPS exposure ${ }^{[6,7,25]}$. These observations suggest that increased intestinal apoptosis and diminished cell proliferation play an important role in LPS-induced intestinal damage. Our previous study showed that enterocyte proliferation is inhibited during endotoxemia and that Ber promotes the enterocyte proliferation in the ileum of endotoxemic mice in an $\mathrm{a}_{2}$-adrenoceptor-independent manner ${ }^{[26]}$. Therefore, we further examined the effect of Ber and $Y$ on enterocyte apoptosis in the ileum in LPS-challenged mice. The results demonstrate that LPS treatment activates caspase- 3 and promotes enterocyte apoptosis in the ileum. Pretreatment with Ber decreases caspase-3 activation and enterocyte apoptosis in the ileum of LPS-challenged mice, and these effects are not antagonized by Y. Other studies have demonstrated an increase in the activity of caspase-3, an increase in the number of apoptotic epithelial cells and a high gut permeability following the intraperitoneal injection of $\mathrm{LPS}^{[27]}$. These findings indicate that the inhibition of enterocyte apoptosis may contribute to the protection of Ber against LPS-induced intestinal injury and that the protective action of Ber is not associated with the $\mathrm{a}_{2}$-adrenoceptor. However, the mechanisms responsible for the reduced enterocyte apoptosis by Ber in endotoxemic mice are unclear. Recent studies have demonstrated that Ber significantly activates the adenosine 5'-monophosphate-activated protein kinase (AMPK) signaling pathway and that AMPK activators protect against tumor necrosis factor-a-induced cardiac cell apoptosis. On the other hand, polymorphonuclear leukocyte transepithelial migration is sufficient by itself to induce intestinal epithelial apoptosis ${ }^{[28-30]}$. Thus, the inhibitory effect of Ber on enterocyte apoptosis in endotoxemia may be related to AMPK activation and the inhibition of leukocyte transepithelial migration, and these questions remain to be investigated.

In addition to apoptosis and hypoplasia, neutrophil infiltration is another important mechanism for intestinal injury during endotoxemia. As the principal circulating phagocyte, neutrophils are the first and most abundant of the leukocytes to arrive at the infection focus ${ }^{[31]}$, and they are ideally suited to eliminate pathogenic bacteria ${ }^{[32]}$. Indeed, neutrophils contain numerous potent tissue-damaging substances, such as reactive oxygen species, bactericidal permeability-increasing proteins and elastases ${ }^{[33]}$, and upon release into the intestinal wall, these substances may contribute to the disruption of intestinal integrity and the increase in macromolecular passage across the epithelial cell lining. In addition, neutrophils can produce cytokines and chemokines that enhance the acute inflammatory response during sepsis ${ }^{[33]}$. Some studies have demonstrated that LPS-associated intestinal leakage in the gut is largely regulated by leukocyte accumulation ${ }^{[16]}$. Thus, we observed the effects of Ber and Y on MPO activity, a useful indicator of neutrophil recruitment, in the ileum of LPS-challenged mice and found that pretreatment with Ber or Ber in combination with Y, but not with Y alone, significantly decreased MPO activity in LPS-challenged mice. This suggests that Ber may inhibit neutrophil recruitment in the ileum during LPS endotoxemia via an $\mathrm{a}_{2}$-adrenoceptor-independent pathway. However, Ber also decreased MPO activity in the ileum in normal control mice, suggesting that MPO activity may not accurately 
reflect neutrophil recruitment under the conditions presented here. Therefore, we determined the amount of MPO in the ileum using a Western blot assay. The results confirmed that pretreatment with Ber significantly decreased the MPO content in the ileum in LPS-challenged mice, an effect that was not reversed by $Y$, suggesting that Ber protects against LPSinduced intestinal injury by inhibiting neutrophil infiltration in an $\mathrm{a}_{2}$-adrenoceptor-independent fashion.

Neutrophil accumulation is thought to be dependent on the formation and activity of CXC chemokines, such as MIP- ${ }^{[16,17]}$. Several studies have shown that LPS-induced MIP-2 expression is involved in I-kB phosphorylation and NF- $\mathrm{kB}$ activation via TLR4 $4^{[15,17,34]}$. A more recent study suggests that Ber may reduce LPS-induced intestinal injury by suppressing the activation of TLR4 and NF-kB in the ileum ${ }^{[10]}$, but a quantitative immunohistochemical assay of TLR4 and NF- $\mathrm{KB}$ activation is needed to confirm this hypothesis. To understand further the signaling pathway involved in the inhibition of neutrophil accumulation induced by Ber in the ileum of LPS-challenged mice, we investigated TLR4 mRNA expression, I-кBa phosphorylation and MIP-2 production in the ileum. The results demonstrate that LPS induced TLR4 mRNA expression, I-kBa phosphorylation and MIP-2 production in the ileum and that these effects were reduced by pretreatment with Ber or Ber in combination with $\mathrm{Y}$, but not with $\mathrm{Y}$ alone. Kessel et al also demonstrated that TLR-4 upregulation might be responsible for the harmful effects of LPS on the intestine $e^{[13]}$. These investigations indicate that pretreatment with Ber inhibits neutrophil infiltration and, in turn, alleviates LPS-induced intestinal injury by suppressing the TLR4-NF-kB-MIP-2 pathway independently of the $\mathrm{a}_{2}$-adrenoceptor.

In conclusion, the results of this study demonstrate that pretreatment with Ber protects against intestinal damage in endotoxemia by reducing neutrophil infiltration and enterocyte apoptosis in an $\mathrm{a}_{2}$-adrenoceptor-independent manner. Ber-induced suppression of neutrophil infiltration in the ileum during endotoxemia is associated with the inhibition of the TLR4-NF-kB-MIP-2 signaling pathway but not with the $\mathrm{a}_{2}$-adrenoceptor. These findings may provide a new therapeutic strategy for the treatment of intestinal injury in sepsis.

\section{Acknowledgements}

We thank Ms Zheng PEIE for her excellent technical assistance. This study was supported by grants from the National Natural Science Foundation of China (grant № 30670826 and 30971191), the Science and Technology Foundation from the Ministry of Education of the People's Republic of China (grant No 207140), the Guangdong Science and Technology Projects (grant No 2008B030301352) and the Leading Academic Discipline Program, 211 Project for Ji-nan University (the 3rd phase).

\section{Author contribution}

Hong-mei LI and Yi-yang WANG performed research, analyzed the data, and drafted the manuscript. Wen-juan CAO, Xiao-hui YU, Ren-bin QI, Chao-feng HU, and Yu-xia YAN performed some of the experiments. Da-xiang LU revised the manuscript. Hua-dong WANG designed the research, obtained the funding and revised the manuscript.

\section{References}

1 Angus DC, Linde-Zwirble WT, Lidicker J, Clermont G, Carcillo J, Pinsky MR. Epidemiology of severe sepsis in the United States: analysis of incidence, outcome, and associated costs of care. Crit Care Med 2001; 29: 1303-10.

2 De-Souza DA, Greene LJ. Intestinal permeability and systemic infections in critically ill patients: effect of glutamine. Crit Care Med 2005; 33: 1175-8.

3 Doig CJ, Sutherland LR, Sandham JD, Fick GH, Verhoef M, Meddings JB. Increased intestinal permeability is associated with the development of multiple organ dysfunction syndrome in critically ill ICU patients. Am J Respir Crit Care Med 1998; 158: 444-51.

4 Deitch EA. The role of intestinal barrier failure and bacterial translocation in the development of systemic infection and multiple organ failure. Arch Surg 1990; 125: 403-4.

5 Pålsson-McDermott EM, O'Neill LA. Signal transduction by the lipopolysaccharide receptor, Toll-like receptor-4. Immunology 2004; 113: 153-62.

6 Sukhotnik I, Mogilner J, Krausz MM, Lurie M, Hirsh M, Coran AG, et al. Oral arginine reduces gut mucosal injury caused by lipopolysaccharide endotoxemia in rat. J Surg Res 2004; 122: 256-62.

7 Sukhotnik I, Agam M, Shamir R, Shehadeh N, Lurie M, Coran AG, et al. Oral glutamine prevents gut mucosal injury and improves mucosal recovery following lipopolysaccharide endotoxemia in a rat. J Surg Res 2007; 143: 379-84.

$8 \mathrm{Kim}$ TS, Kang BY, Cho D, Kim SH. Induction of interleukin-12 production in mouse macrophages by berberine, a benzodioxoloquinolizine alkaloid, deviates $\mathrm{CD}^{+} \mathrm{T}$ cells from a Th2 to a Th1 response. Immunology 2003; 109: 407-14.

9 Li F, Wang HD, Lu DX, Wang YP, Qi RB, Fu YM, et al. Neutral sulfate berberine modulates cytokine secretion and increases survival in endotoxemic mice. Acta Pharmacol Sin 2006; 27: 1199-205.

10 Zhang Q, Piao XL, Piao XS, Lu T, Wang D, Kim SW. Preventive effect of Coptis chinensis and berberine on intestinal injury in rats challenged with lipopolysaccharides. Food Chem Toxicol 2010; 49: 61-9.

11 Hui KK, Yu JL, Chan WF, Tse E. Interaction of berberine with human platelet alpha 2 adrenoceptors. Life Sci 1991; 49: 315-24.

12 Zhang HQ, Wang HD, Lu DX, Qi RB, Wang YP, Yan YX, et al. Berberine inhibits cytosolic phospholipase A2 and protects against LPS-induced lung injury and lethality independent of the alpha 2-adrenergic receptor in mice. Shock 2008; 29: 617-22.

13 Kessel A, Toubi E, Pavlotzky E, Mogilner J, Coran AG, Lurie M, et al. Treatment with glutamine is associated with down-regulation of Tolllike receptor-4 and myeloid differentiation factor 88 expression and decrease in intestinal mucosal injury caused by lipopolysaccharide endotoxaemia in a rat. Clin Exp Immunol 2007; 151: 341-7.

14 De Plaen IG, Tan XD, Chang H, Wang L, Remick DG, Hsueh W. Lipopolysaccharide activates nuclear factor kappa $B$ in rat intestine: role of endogenous platelet-activating factor and tumour necrosis factor. Br J Pharmacol 2000; 129: 307-14.

15 Ohtsuka Y, Lee J, Stamm DS, Sanderson IR. MIP-2 secreted by epithelial cells increases neutrophil and lymphocyte recruitment in the mouse intestine. Gut 2001; 49: 526-33.

16 Mangell P, Mihaescu A, Wang Y, Schramm R, Jeppsson B, Thorlacius $H$. Critical role of P-selectin-dependent leukocyte recruitment in endotoxin-induced intestinal barrier dysfunction in mice. Inflamm Res 
2007; 56: 189-94.

17 De Plaen IG, Han XB, Liu X, Hsueh W, Ghosh S, May MJ. Lipopolysaccharide induces $\mathrm{CXCL2/macrophage} \mathrm{inflammatory} \mathrm{protein-2}$ gene expression in enterocytes via NF-kappa B activation: independence from endogenous TNF-alpha and platelet-activating factor. Immunology 2006; 118: 153-63.

18 Oktar BK, Gulpinar MA, Bozkurt A, Ghandour S, Cetinel S, Moini H, et al. Endothelin receptor blockers reduce //R-induced intestinal mucosal injury: role of blood flow. Am J Physiol Gastrointest Liver Physiol 2002; 282: G647-G55.

19 Song J, Wolf SE, Herndon DN, Wu XW, Jeschke MG. Second hit post burn increased proximal gut mucosa epithelial cells damage. Shock 2008; 30: 184-8.

20 Yasuda T, Takeyama Y, Ueda T, Shinzeki M, Sawa H, Nakajima T, et al. Breakdown of intestinal mucosa via accelerated apoptosis increases intestinal permeability in experimental severe acute pancreatitis. J Surg Res 2006; 135: 18-26.

21 Zayat M, Lichtenberger LM, Dial EJ. Pathophysiology of LPS-induced gastrointestinal injury in the rat: role of secretory phospholipase A2. Shock 2008; 30: 206-11.

22 Yuan ZQ, Peng YZ, Li XL, Huang YS, Yang ZC. Induction of heat shock protein 70 by sodium arsenite attenuates burn-induced intestinal injury in severe burned rats. Burns 2008; 34: 247-53.

23 Croci T, Landi M, Galzin AM, Marini P. Role of cannabinoid CB1 receptors and tumor necrosis factor-alpha in the gut and systemic anti-inflammatory activity of SR 141716 (rimonabant) in rodents. Br J Pharmacol 2003; 140: 115-22.

24 Wang YY, Li HM, Wang HD, Peng XM, Wang YP, Lu DX, et al. Pretreatment with berberine and yohimbine protects against lippolysaccharide-induced myocardial dysfunction via inhibition of cardiac I-kB $\alpha$ phosphorylation and apoptosis in mice. Shock 2011; 35: 322-8.

25 Sukhotnik I, Shehadeh N, Rothem L, Lurie M, Mogilner J, Shiloni E, et al. Oral insulin up-regulates Toll-like receptor 4 expression and enhances intestinal recovery following lipopolysaccharide-induced gut injury in a rat. Dig Dis Sci 2008; 53: 1231-9.

26 Li HM, Wang YY, Wang HD, Cao WJ, Jiang JW, Hu CF, et al. Effect of berberine and yohimbine on impaired enterocyte proliferation and intestinal injury in LPS-challenged mice. Chin J Pathophysiol 2010; 26: 941-6.

27 Alscher KT, Phang PT, McDonald TE, Walley KR. Enteral feeding decreases gut apoptosis, permeability, and lung inflammation during murine endotoxemia. Am J Physiol Gastrointest Liver Physiol 2001; 281: G569-G76.

28 Lu DY, Tang CH, Chen YH, Wei IH. Berberine suppresses neuroinflammatory responses through AMP-activated protein kinase activation in BV-2 microglia. J Cell Biochem 2010; 110: 697-705.

29 Kewalramani G, Puthanveetil P, Wang F, Kim MS, Deppe S, Abrahani A, et al. AMP-activated protein kinase confers protection against TNF- $\alpha$ induced cardiac cell death. Cardiovasc Res 2009; 84: 42-53.

30 Le'Negrate G, Selva E, Auberger P, Rossi B, Hofman P. Sustained polymorphonuclear leukocyte transmigration induces apoptosis in T84 intestinal epithelial cells. J Cell Biol 2000; 150: 1479-88.

31 Lekstrom-Himes JA, Gallin J. Immunodeficiency diseases caused by defects in phagocytes. N Engl J Med 2000; 343: 1703-14.

32 Seely AJ, Pascual JL, Christou NV. Science review: Cell membrane expression (connectivity) regulates neutrophil delivery, function and clearance. Crit Care 2003; 7: 291-307.

33 Guo RF, Riedemann NC, Sun L, Gao H, Shi KX, Reuben JS, et al. Divergent signaling pathways in phagocytic cells during sepsis. J Immunol 2006; 177: 1306-13.

34 De Filippo K, Henderson RB, Laschinger M, Hogg N. Neutrophil chemokines $K C$ and macrophage-inflammatory protein-2 are newly synthesized by tissue macrophages using distinct TLR signaling pathways. J Immunol 2008; 180: 4308-15. 